\title{
L'arrivée des migrants sur les écrans de cinéma italiens
}

L'arrivo dei migranti sugli schermi cinematografici italiani

Foreign migrants in Italian cinema

Isabelle Felici

\section{(2) OpenEdition}

\section{Journals}

Édition électronique

URL : http://journals.openedition.org/cei/545

DOI : $10.4000 /$ cei.545

ISSN : 2260-779X

\section{Éditeur}

UGA Éditions/Université Grenoble Alpes

Édition imprimée

Date de publication : 15 mars 2012

Pagination : 235-244

ISBN : 978-2-84310-222-6

ISSN : $1770-9571$

\section{Référence électronique}

Isabelle Felici, «L'arrivée des migrants sur les écrans de cinéma italiens », Cahiers d'études italiennes

[En ligne], 14 | 2012, mis en ligne le 15 septembre 2013, consulté le 26 mars 2021. URL : http:// journals.openedition.org/cei/545 ; DOI : https://doi.org/10.4000/cei.545 


\title{
L'ARRIVÉE DES MIGRANTS SUR LES ÉCRANS DE CINÉMA ITALIENS
}

\author{
Isabelle Felici \\ Université Montpellier 3
}

Il est aisé de dater l'arrivée des migrants sur les écrans de cinéma italiens avec Pummarò ${ }^{1}$ de Michele Placido, produit en 1989 et sorti en I990. Notre premier regard s'est porté sur ce film vingt ans après sa sortie, alors que de nombreuses autres images, toutes plus récentes, mettant en scène des immigrés en Italie ${ }^{2}$, l'avaient nourri. Le spectateur garde, gravé dans sa mémoire, le premier regard qu'il a eu sur un film ${ }^{3}$ et tout autant indélébile est sa première impression, utile pour l'élaboration d'une hypothèse de lecture ${ }^{4}$ : en dehors de toute considération esthétique, Pummarò nous est apparu plus ouvert, plus éclairé, moins emprunté et moins embarrassé, moins pudique même, pour aborder la question migratoire, que les films des décennies suivantes. Le spectateur y est immergé dans les problématiques de l'immigration, qu'il suit de l'intérieur, des problématiques qui évoluent au fur et à mesure qu'avance le récit. On suit en effet le protagoniste, Kwaku, dans sa remontée de l'Italie depuis le port de Naples, où il débarque d'un bateau qui l'a transporté clandestinement, jusqu’à Vérone, puis en Allemagne, à Francfort, où le conduit l'enquête qu'il mène pour retrouver les traces de son frère. Tout est montré du point de vue du protagoniste évoluant dans son milieu, sans jugement, avec une formidable capacité d'écoute et d'adaptation de la part du metteur en scène.

I. M. Placido, Pummarò, I990, avec T. A. K. Amenya, P. Villoresi, F. Interlenghi, J. Williams, G. Scala, DVD Cecchi Gori Home Video.

2. Pour une liste de films sur le sujet, voir S. Cincinelli, I migranti nel cinema italiano, Rome, Kappa, 2009.

3. Voir le souvenir d'enfance d'Italo Calvino raconté dans "Autobiografia di uno spettatore ", rédigé en I974, pour la préface d'un livre de Federico Fellini et repris dans le recueil La strada di San Giovanni, Milan, Mondadori.

4. Voir par exemple J. Aumont et M. Marie, L'Analyse des films, Paris, Nathan, 1989, p. 49. 
Si Placido, qui signe ici son premier long-métrage en tant que réalisateur, arrive d'emblée à ce résultat, c'est qu'il se situe dans la lignée des films italiens ayant traité les questions migratoires à partir du vécu des Italiens partis à l'étranger. De façon implicite contrairement à d'autres films plus tardifs, ce vécu est naturellement transposé à celui d'autres immigrés, en l'occurrence des Africains arrivés en Italie. Bien que ce phénomène migratoire soit encore nouveau pour l'Italie de la fin des années quatre-vingt, Placido le met en images sans rupture par rapport aux films antérieurs qui ont abordé le sujet. La rupture est en revanche très nette par rapport aux films postérieurs.

\section{Pummarò et ses « frères »}

Contrairement à Lamerica de Gianni Amelio's, l'autre film marquant pour les questions migratoires, sorti quelques années après, en 1994, Pummarò se passe entièrement en Italie; seul le bref épilogue se déroule à Francfort. Le voyage du protagoniste arrivant d'Afrique est à peine ébauché, pour des nécessités narratives. Grâce à une caméra placée sur le bateau lui-même, les images qui précèdent le générique font allusion au mouvement et au déplacement.

Malgré des scènes très réussies, Pummarò est sûrement moins abouti du point de vue esthétique que Lamerica, qui est déjà devenu un monument du cinéma italien et qui, comme tout monument, souffre parfois de jugements et de classements trop rapides et passe-partout. Certains font en effet de Lamerica une épopée de l'émigration italienne ou, de façon tout aussi réductrice, un documentaire fiction sur l'immigration albanaise en Italie. Le premier raccourci trouve son origine dans le parallèle, explicite notamment dans le titre et dans les images finales, entre émigration italienne et immigration en Italie : suite à un concours de circonstances, voyagent dans le même bateau des Albanais au regard empli du " rêve d'Italie " et Michele/Spiro, ce vieux soldat de l'armée mussolinienne devenu amnésique, persuadé qu'il va débarquer à New-York. Le second raccourci n'est guère plus pertinent puisque, contrairement à Kwaku, qui met le pied sur le sol italien dès les premières minutes du film de Placido, les réfugiés albanais d'Amelio sont montrés uniquement au cours de leur voyage migratoire, qui occupe toute la dernière partie du film (environ le tiers), après l'infâme escroquerie et la déchéance du protagoniste; mais on ne les

5. G. Amelio, Lamerica, 1994, avec M. Placido et E. Loverso, DVD Cecchi Gori Home Video. 
voit ni descendant du bateau ni s'installant dans leur nouvelle vie. Les dernières images de Lamerica, d'où est de plus en plus absent le protagoniste qui a enfin cessé de voir ses compagnons de voyage à travers le filtre déformant de ses lunettes de soleil de marque, font défiler une galerie de visages d'hommes, de femmes et d'enfants, au regard plein d'espoir, confiants en ce qu'ils vont trouver à leur arrivée. Par ces gros plans insistants, Gianni Amelio conduit ses spectateurs à retirer à leur tour leur filtre déformant, en faisant explicitement appel, grâce au vieil homme amnésique, à leur passé migratoire refoulé; il tente, en d'autres termes, de leur rafraîchir la mémoire $^{6}$ :

Avete visto quanta gente! Io non credia che simbarcavano tutti. [...] Paesà, ma voi sapete parlare americano? Io non saccio manco litaliano. Che dicete, ci danno lo stesso un lavoro? Sugnu stanco, ma voglio stare sveglio quando arriviamo a Nuova York ${ }^{7}$.

Une autre représentation cinématographique des arrivées massives d'Albanais en Italie est donnée par Nanni Moretti dans une scène d'Aprile, sorti en 1998. La scène ne dure que trois minutes dans ce film témoin des bouleversements que connaît l'Italie lors de la dernière décennie du $\mathrm{xx}^{\mathrm{e}}$ siècle. Elle prend appui sur le naufrage d'un bateau albanais torpillé par la marine italienne ${ }^{8}$ avant son arrivée sur les plages de l'Adriatique et illustre le désarroi de l'Italie face à ces flux migratoires de plus en plus massifs. Irrité de se rendre compte qu'il est incapable de sortir de ses préoccupations du moment pour poser des questions pertinentes à des Albanais qu'il rencontre dans un centre d'accueil, le réalisateur/personnage s'en prend aussi à la classe politique italienne - le centre-gauche est alors au gouvernement - qui selon lui n'a pas su accueillir ces réfugiés :

Che domande da deficienti. Proprio fuori luogo... E poi il fatto che in questi giorni qui in Puglia non sia venuto nemmeno un dirigente della sinistra è il sintomo della loro assenza politica, ma soprattutto della loro assenza umana ${ }^{9}$.

Cette fois, le spectateur dépasse le moment du voyage, mais ne va pas au-delà du moment de l'arrivée dans le pays d'accueil. Aucun des films

6. C'est la même démarche qu'adopte Ettore Scola dans un épisode de Gente di Roma, avec davantage de cynisme, comme si l'entreprise lui semblait désespérée : le propriétaire d'un bar s'épanche sur le souvenir de son père mort à la mine à Marcinelle et, dans la même conversation, déverse son fiel à l'encontre d'un client d'origine africaine.

7. Nous transcrivons d'après les dialogues du film.

8. "Nel tardo pomeriggio del 28 marzo 1997, un Venerdi santo, la nave della marina militare italiana Sibilla speronò il battello albanese Kater I Rades, che si trovava ancora in acque internazionali, ma che cercava di avvicinarsi alle coste pugliesi. I morti furono 58, tra cui numerosi bambini. " (<http://archiviostorico.corriere.it/ 2000/ dicembre/I7/Albanesi_morti_Otranto_dall_Italia_co_o_oor2179335.shtml>)

9. Nous transcrivons d'après les dialogues du film, DVD Studio Canal. 
italiens qui ont suivi Pummarò n'a montré, comme l'a fait Placido, des immigrés installés, ayant trouvé un moyen de subsistance qui n’a pas forcément à voir avec la délinquance ou la prostitution. Dans Pummarò, les immigrés ont déjà appris à se mouvoir avec facilité dans la société italienne et à jongler avec ses contradictions; les enfants vont à l'école, les parents travaillent dans des usines italiennes, certains vont à l'église. Il faut attendre 2007 pour retrouver un autre immigré non clandestin, non délinquant, de surcroît bien établi dans le tissu social italien et qu’on voit évoluer dans son milieu familial et culturel : c'est Hassan, dans le film de Carlo Mazzacurati, La giusta distanza ${ }^{\mathrm{IO}}$.

Le désarroi des Italiens à l'égard des immigrés apparaît aussi dans des films qui ne traitent pas directement de la question migratoire et il est encore plus perceptible lorsqu' on procède à une comparaison avec Pummarò. Dans Caterina va in città, 2002, Paolo Virzì montre sa jeune protagoniste dans un bus romain. La caméra ne cesse d'errer de son visage aux yeux curieux et étonnés à celui des passagers, surtout d'origine étrangère, s'arrêtant en particulier sur des femmes africaines. Le contraste est frappant avec la scène de Pummarò au cours de laquelle le protagoniste rencontre lui aussi des jeunes filles dans un bus romain : les personnages sont filmés en plans rapprochés, donnant l'impression au spectateur d'être un passager du même autobus, et la scène est pour tous l'occasion d'un échange, certes fugitif, mais souriant et détendu.

Ce désarroi, perceptible dans tous les films sortis après 1994, prend les formes les plus diverses, pouvant se transformer en véritable soif de connaître et de comprendre. Le premier long-métrage de Vincenzo Marra, Tornando a casa, 200I, est le meilleur exemple d'une incompréhension qui se transforme en attirance et qui prend, à travers les choix du protagoniste, surtout dans la scène finale, des proportions inattendues.

Même Nuovomondo d'Emanuele Crialese, 2006, se situe dans une démarche de redécouverte, par le cinéma italien, du thème de l'émigration italienne pourtant régulièrement abordé depuis les origines ${ }^{\text {II }}$. Le réalisateur choisit de privilégier le thème du départ (depuis le moment où se prend la décision de partir, jusqu'au voyage lui-même et au passage à Ellis Island), dans une démarche proche de celle d'Amelio et Scola (pour les séquences que nous avons déjà citées), qui vient rappeler au public italien les enjeux

Io. Carlo Mazzacurati avait abordé le thème de l'immigration avec Vesna va veloce en 1998 . Il y met en scène une jeune Tchèque qui profite d'un voyage touristique pour rester en Italie, où elle survit en se prostituant.

II. On trouvera une liste de films italiens sur l'émigration dans J. A. Gili, «L'émigration italienne au cinéma ", dans L. Teulières (dir.), Au cour des racines et des hommes, Radici, hors-série, juillet 2007. 
de son passé migratoire, réactivés par la présence d'immigrés en Italie. Dans cette logique, il n'est pas étonnant que Crialese, qui a aussi traité la question migratoire du point de vue de l'immigré italien dans son pays d'accueil (Once we were strangers, 1997), ait choisi de mettre en scène dans son nouveau film, Terraferma, en cours de tournage au moment où nous écrivons, une femme africaine qui débarque clandestinement sur une île proche de la Sicile.

En parcourant à rebours, à partir de Pummarò, les chemins du cinéma italien, on trouve plusieurs films qui racontent eux aussi les vicissitudes de l'installation dans le nouvel environnement : Good morning Babilonia, 1986, de Paolo et Vittorio Taviani, (qui met également en place les mécanismes du départ et esquisse le voyage transatlantique) et surtout Pane e cioccolata de Franco Brusati, 1974. Sur la même problématique de l'installation, on peut remonter à Rocco et ses frères (Luchino Visconti, 196I), Profession magliari (Francesco Rosi, 1959), jusqu'à 1934 et Toni de Jean Renoir, aux origines du néo-réalisme ${ }^{\mathrm{I} 2}$.

\section{Pummarò néo-réaliste}

Si l'on en croit ses propos, dans un entretien qu'il a donné au moment de la sortie du film en DVD, Michele Placido a voulu rendre hommage au cinéma néoréaliste italien, qui a été une source d'inspiration pour Pummarò $^{13}$. Pour illustrer sa démarche, il cite la participation au casting de Franco Interlenghi, qui a joué enfant dans Sciuscià (Vittorio De Sica, 1946).

Il raconte aussi comment l'idée de ce film est née lorsque, en revenant d'une journée à la plage, il a croisé le chemin d'Africains, dont l'un était médecin comme Kwaku, employés à ramasser des tomates. C'est exactement la démarche de Giuseppe De Santis qui, revenant d'un séjour à Paris, a croisé le chemin des mondine et s'est inspiré de cette rencontre pour tourner Riso amaro (1949).

I2. Même si le film a souvent été présenté ainsi, Renoir a récusé le fait que Toni puisse être considéré comme "précurseur des films néo-réalistes italiens ", avançant le fait qu’il s'était " efforcé de ne pas être dramatique " et qu'il n'avait pas utilisé le doublage, mais bien le son en prise directe (J. Renoir, Ma vie et mes films, Paris, Flammarion, 1974, p. I4O-I4I). Dans un texte plus ancien (1956), le cinéaste trouve cependant une parenté dans sa démarche et celle du néo-réalisme, lorsqu'il s'agit de filmer les « phases d'un conflit sans que les êtres humains inconsciemment entraînés dans cette action s'en soient aperçus. [...] Plus tard, le néo-réalisme italien devait pousser le système jusqu’à la perfection " (J. Renoir, Écrits (I926-I97I), Belfond (1974), I993, p. 3IO).

I3. P. Toccafondi et T. Santi, «Il mio debutto neorealista. Intervista a Michele Placido », supplément au DVD de Pummarò, Cecchi Gori Home Video. 
L'hommage au néo-réalisme tient certes aussi à l'emploi d'acteurs nonprofessionnels, "ramassés dans la rue » dit Placido, au tournage en décors réels, aux nombreuses scènes à dimension documentaire. Mais surtout, en visionnant Pummarò, le spectateur est renvoyé aux images de films de l'après-guerre, premier entre tous Paisà. Pummarò est un film à épisodes géographiques ${ }^{14}$ : le premier chapitre, juste après l'introduction et l'arrivée sur le port de Naples, se déroule à Civitella, dans la province de Benevento, dans le milieu des ramasseurs de tomates encadrés par des associations mafieuses, le second à Rome, dans les quartiers de la prostitution, le troisième à Vérone, dans une famille d'Africains bien installée professionnellement et socialement; comme on l'a vu, l'épilogue a lieu à Francfort. Le spectateur fait aussi le rapprochement entre les deux films à cause du personnage principal : un grand Noir qui part à la conquête de la péninsule et qui, de plus, vient mettre l'Italie face à ses réalités, misères et contradictions.

C'est grâce à cette intimité avec le passé que Placido gagne en modernité et peut saisir, du premier coup, ses personnages immigrés en Italie dans toute leur profondeur. Il est troublant de voir combien le parallèle est parfait entre le parcours d'Antonio Canova, le Toni de Jean Renoir que nous venons d'évoquer, et celui de Kwaku Kwalaturé, qui forme un personnage double avec son frère Giobbe. L'économie des deux récits est très proche, avec un voyage à peine esquissé et une arrivée de plain pied dans les réalités du pays d'accueil. On est très vite interpellé par le fait que Toni et Kwaku parlent la langue du pays dès qu'ils posent le pied dans leur nouvel univers. C'est plausible dans les deux cas puisque Toni est un « habitué » et n'en est pas à son premier séjour en Provence et que Kwaku a préparé son voyage, en prenant des cours d'italien. Les deux personnages sont aussi tous deux en quête de leur double, un double féminin pour Toni et un frère pour Kwaku; la quête se conclut à chaque fois par une mort violente, pour une «bonne" cause.

Le parallèle continue sur le plan technique, par exemple avec l'utilisation de la musique. Dans Pummarò, l'acteur qui interprète le rôle de Kwaku est musicien ${ }^{15}$ et prête son talent à son personnage dans plusieurs scènes où la musique diégétique est reliée par celle que signent Lucio Dalla et Mauro Malvasi. C’est le guitariste et chanteur Paul Bozzi qui est crédité de la bande sonore de Toni; il tient aussi le rôle d'un des personnages, ouvrier comme Toni, et interprète plusieurs airs tout au long du film, en

I4. Le parallèle serait également à faire avec Il cammino della speranza de Pietro Germi (I950), où on suit un groupe d'émigrants qui remonte toute la péninsule, depuis la Sicile, jusqu’à la frontière française.

I5. Voir les propos de M. Placido dans "Il mio debutto neorealista ", cité. 
français, en italien et en corse surtout, des chansons qui sont une des clefs d'interprétation du film et de sa dimension tragique :

Le sujet du film est tiré d'un fait divers qui s'est réellement passé dans un coin du midi de la France resté suffisamment sauvage pour permettre une photographie dramatique. Cette région est habitée principalement par des immigrants d'origine italienne, mi-ouvriers, mi-paysans. Chez ces déracinés les passions sont vives et les hommes qui me servirent de modèle pour Toni m'ont semblé traîner derrière eux cette atmosphère lourde, signe du destin fatal des héros de tragédie, voire de chanson populaire ${ }^{16}$.

Les deux films sont aussi chacun pionnier à leur façon. Pummarò est le premier long-métrage sur le sujet de l'immigration en Italie et, bien que l'immigration italienne en France soit un phénomène déjà ancien quand Renoir commence son film ${ }^{17}$, il n'a pas inspiré les cinéastes français avant lui ${ }^{18}$. Les deux films sont aussi le reflet de la diversité migratoire. Dans l'Italie des années quatre-vingt qui se dessine dans Pummarò, Kwaku croise d'autres immigrés, marocains notamment. Quant à Renoir, il fait de cette diversité un arrière-plan permanent de son film, qui se déroule en Provence dans l'entre-deux-guerres ${ }^{19}$. En 1934, la " fusion des races ${ }^{20}$ " a déjà commencé, non sans heurts comme le rappellent quelques répliques dans les premières minutes du film : les Piémontais qui débarquent du train (mais on mentionne aussi des Espagnols « arrivés quelque temps plus tôt à Miramas ") sont annoncés comme " ces étrangers qui viennent nous lever le pain ", la réplique étant placée dans la bouche d'un Turinois et d'un Barcelonais, à peine moins récemment arrivés. C'est aussi un Espagnol qui exprime sa méfiance envers les étrangers que les grands travaux attirent dans le pays : "surtout les sidis. Oh, je les aime pas ceux-là. On peut pas

I6. Lettre de Jean Renoir, datée d'octobre 1934, destinée aux directeurs de salle. Le texte, publié dans un article de Comoedia du 8 février 1935, est repris dans Jean Renoir, Écrits, ouvr. cité, p. 308.

17. Le film est présenté comme " une histoire vraie racontée par Jean Renoir, d'après la documentation recueillie par Jacques Levert ". Jacques Levert (de son vrai nom Jacques Mortier), à l'époque commissaire de police de la ville de Martigues (Jean Renoir, Ma vie et mes films, ouvr. cité, p. I40) projetait de faire un livre de ce fait divers; le livre n'est jamais sorti, peut-être à cause du maigre succès remporté par le film à sa sortie en 1935. De Jacques Levert, on pourra lire en revanche le roman policier Le singe rouge, Paris, S.E.P.E., 1946.

I8. J.-C. Vegliante, "Appunti sulla rappresentazione filmica degli italiani in Francia ", ASEI, mai 2008 (<http://www.asei.eu/index.php?option=com_content\&view=article\&id=I66:appunti-sulla-rappresentazionefilmica-degli-italiani-in-francia\&catid=65:articoli\&Itemid=250>). Voir aussi la rubrique Cinémigration dans la revue du CEDEI, La Trace (les sommaires sont disponibles sur <http://cedei.univ-parisi.fr/tracethem.htm>) ainsi que D. Serceau, «Les Italiens les premiers. 1934: Un film précurseur, Toni, de Jean Renoir » (Cinémas de l'émigration, Cinémaction, $\mathrm{n}^{\circ}$ 8, 1979).

19. À propos de Toni, Émile Témime souligne la diversité des personnages (des Italiens, un Belge, des Espagnols), " qui marque précisément à Marseille l'immigration de l'entre-deux-guerres » (É. Témime, "Toni, une image de l'émigration italienne sur le littoral méditerranéen ", Marseille, n 173-174, 1995).

20. Nous reprenons l'expression de la didascalie qui introduit le film : "L'action se passe dans le midi de la France, en pays latin, là où la nature, détruisant l'esprit de Babel, sait si bien opérer la fusion des races. ” 
savoir ce qu'ils pensent ${ }^{21}$. On relève moins souvent la présence corse, mise pourtant au premier plan par Jean Renoir à travers les chansons populaires qui, on l'a vu, rythment tout le film, pour la plupart des chansons corses ${ }^{22}$, et à travers le personnage de Primo, ouvrier et musicien corse "émigré " lui aussi en Provence.

Mais ce qui rapproche le plus les deux films, c'est le parcours d'installation, certes manquée, à une nouvelle condition auquel on assiste dans les deux cas. Ne manquent pas les scènes familiales, dans lesquelles Toni et Kwaku sont d'ailleurs tous deux spectateurs.

Le travail est un thème récurrent dans les deux films : " mon pays c'est celui qui me fait bouffer ", "ça sent le pétrole, mais ça sent le boulot aussi ", disent les Italiens en arrivant à Martigues. Nombreuses sont les scènes tournées aux champs ou à l'usine, à la mine, sur les voies ferrées... Comme les Espagnols et les Italiens de Toni, les Africains de Pummarò construisent leur pays de demain.

\section{Italia dolce casa}

Les Africains sont donc bien montrés chez eux, en Italie, même si le réalisateur de Pummarò semble éprouver un malin plaisir à dérouter le spectateur en lui montrant des images qui peuvent sembler exotiques, comme tout droit sorties d'un documentaire de voyage : par exemple ces buffles ou ces files de camion partant avant l'aube, comme en expédition.

Ces images ancrent au contraire le film dans la réalité italienne ${ }^{23}$ et, pour le premier épisode, dans une des provinces où l'on produit depuis des siècles de la mozzarella à partir de lait de bufflonne.

Le film se caractérise ainsi par une série de décalages qui viennent bouleverser les idées reçues. Placido rapporte qu'on a voulu y voir des exagérations grimaçantes, dignes de la comédie à l'italienne. C'est pourtant de la lecture de faits divers qu'ont été tirées les scènes les plus aberrantes : le cimetière-dortoir dont les emplacements vides servent de lit, le wagon

2I. Nous transcrivons d'après les dialogues du film.

22. Voir le minutieux découpage effectué par C. Peyrusse et L. Borger, sous la direction de C. Beylie, et publié par L'Avant-scène cinéma, $\mathrm{n}^{\circ}$ 25I-252, 1980, p. 2I et suiv.

23. Vingt ans après le film, les conditions de travail des ramasseurs de tomates continuent à être régulièrement dénoncées, par exemple le 28 juillet 2009, lorsque Le Monde en ligne et l'AFP se font le relais des propos de l'Organisation internationale pour les migrations selon lesquels des « immigrés sont exploités dans des conditions "indignes" dans le sud de l'Italie " (<http://www.lemonde.fr/europe/article/2009/07/28/des-immigres-exploitesdans-des-conditions-indignes-dans-le-sud-de-l-italie_I223670_32I4.html\#ens_id=I2I4846>). 
désaffecté utilisé comme restaurant, hôtel et même salle d'accouchement, à deux pas d'une grande gare romaine.

Ces décalages sont sans doute également dus au fait que le scénario s'est enrichi, au moment du tournage, des sensations et de la biographie de l'acteur principal, Thywill A. K. Amenya qui joue le rôle de Kwaku. Le spectateur perçoit ainsi du point de vue du personnage d'origine africaine les effets des visions stéréotypées des Italiens, et des Européens en général, sur l'Afrique. C'est aussi en même temps que lui qu'on s'étonne, ou qu'on se réjouit, des réactions tour à tour ingénues, méchantes, admiratives, amicales : non, en Afrique, Kwaku n'habitait pas dans une case; il a fait de longues études et, passant devant le balcon de Juliette à Vérone, il déclame de mémoire Shakespeare dans le texte, sans qu'il y ait là d'incongruité puisqu'il vient du Ghana où la langue officielle est l'anglais. C'est lui, médecin, qui vient en aide à la jeune toxicomane, italienne, qui accouche dans le wagon désaffecté dont il vient d'être question. Il entre sans difficulté dans le monde du travail, dans une fonderie à Vérone, où il reçoit des marques de solidarité et de sympathie, à la cantine et à l'atelier, tout en ayant à supporter des manifestations d'animosité, par exemple de la part d'un ancien ouvrier qui confond son ressentiment face à l'échec de la lutte qu'il a menée toute sa vie avec la crainte que provoque en lui l'arrivée des ouvriers africains dans son secteur d'activité.

Malgré les obstacles, Kwaku commence à s'installer dans une nouvelle vie, partageant les préoccupations d'une famille africaine : on montre avec insistance des parents soucieux du travail scolaire de leurs enfants, qui ne jugent pas utile de leur enseigner leur langue africaine; ces enfants sont pleins de projets, comme cette petite fille qui, dans un assez long monologue qui permet d'apprécier sa maîtrise du dialecte de Vénétie, annonce qu' elle sera policière ou infirmière ${ }^{24}$. Kwaku entreprend même de s'installer sentimentalement avec une Italienne, mais les violences morales et physiques qu'ils subissent tous deux, la pression sociale, et aussi la quête qu'il doit reprendre pour retrouver son frère, viennent mettre un terme à une relation qui n'avait guère d'avenir : Kwaku avait été mis en garde dès le début, quand on lui avait rapporté l'histoire d'un autre immigré :

- Questo è finito.

- Come finito?

24. On notera que ces métiers diffèrent un peu des étiquettes derrière lesquelles, même avec les meilleures (?) intentions, on enferme l'avenir des migrants en Italie, les vouant uniquement à des métiers subalternes. Voir par exemple le spot diffusé en décembre 2009 : <http://www.youtube.com/user/BlacksOutChannel\#p/a> et repris notamment dans le cadre de la « journée sans immigrés " du $\mathrm{I}^{\mathrm{er}}$ mars $20 \mathrm{O} \mathrm{O}$. 
- L'hanno ammazzato. È morto per amore.

- Per amore?

- Faceva l'amore con una ragazza di queste parti. Ed è finito sotto un camion sulla Domiziana.

On le voit, la tragédie shakespearienne a encore de grands jours devant $e e^{25}$. Placido raconte, dans l'entretien déjà cité, comment on a parfois refusé de projeter le film à cause des scènes d'amour entre une Blanche et un Noir ${ }^{26}$, perçues comme scandaleuses car selon lui la société italienne n'est " historiquement pas prête » à affronter le phénomène migratoire ${ }^{27}$.

Vingt ans après, son film continue à transmettre un message humaniste et intemporel, montrant l'inévitable bouleversement qu'apportent ces nouveaux arrivants aux sociétés qui les accueillent, lesquelles sont souvent loin de se rendre compte des richesses humaines, culturelles, pour ne pas parler des retombées économiques, que renferme leur bagage. $\mathrm{Si}$, comme on le sait grâce à d'autres expériences migratoires, l'installation est inévitable au fil du temps, elle ne va pas jamais de soi. Ainsi Kwaku est-il averti dès le début que son chemin sera semé d'embûches : la première fontaine vers laquelle il se dirige, dans les premières minutes du film, est une fontaine sans eau, justement, lui explique-t-on, parce qu'on veut empêcher "les personnes comme lui » de se laver. La «leçon » lui arrive en condensé : il devra se battre pour survivre et garder sa dignité.

Parce qu'il se situe dans une tradition migratoire, ce film est le premier en Italie, et pour l'instant le plus puissant, à relever sans fausse note la mesquinerie de nos sociétés occidentales qui seules peuvent inventer une punition qu'ailleurs on ne réserverait pas à son pire ennemi, le priver d'eau, source de vie.

25. Citons aussi le surprenant Rometta e Giulieo de Gangbo Jadelin Mabiala, écrivain italien d'origine congolaise, publié par Feltrinelli en 200 .

26. Les scènes de prostitution ne semblent pas choquer autant. Faut-il penser, comme cela a été relevé, que "seule la relation sexuelle interethnique gratuite est dérangeante, contrairement à la relation sexuelle tarifée "? (B. Le Gouez, "Pummarò : la "saison de l'or rouge". Ramasseurs de tomates dans l'Italie des mafias ", dans Tomate et Chocolat : usages alimentaires et créolisation culturelle, Paris, Michel Houdiard éditeur, 2008, p. 72-86.) Notons que le thème a été repris au cinéma en 2007 dans une comédie bourgeoise de C. Comencini, Bianco e nero.

27. Les déclarations, actions, manifestations... qui illustrent ou dénoncent cet état de fait sont nombreuses. Citons l'une des plus récentes, celle de l'écrivain Pap Khouma, d'origine sénégalaise, qui a récemment relancé le débat en racontant, à partir de ses déboires quotidiens, comment les institutions italiennes relaient les pratiques discriminatoires (<http:/www.lemonde.fr/europe/article/20Io/oi/o2/en-italie-le-temoignage-de-pap-khoumablac k-italien-lance-un-debat-sur-le-racisme_I2867I5_32I4.html>). 